\title{
Fundamental Cosmology with the E-ELT
}

\author{
C. J. A. P. Martins ${ }^{1} \dagger$, A. C. O. Leite ${ }^{1,2}$ and P. O. J. Pedrosa $a^{1,2}$ \\ ${ }^{1}$ Centro de Astrofísica, Universidade do Porto, Rua das Estrelas, 4150-762 Porto, Portugal \\ email: Carlos.Martins@astro.up.pt \\ ${ }^{2}$ Faculdade de Ciências, U. Porto, Rua do Campo Alegre, 4150-007 Porto, Portugal
}

\begin{abstract}
The evidence for the acceleration of the universe shows that canonical theories of cosmology and particle physics are incomplete, and that new physics is out there, waiting to be discovered. Forthcoming high-resolution ultra-stable spectrographs will play a key role in this quest for new physics. Here we focus on astrophysical tests of the stability of nature's fundamental couplings, and by taking existing VLT data as a starting point we discuss how forthcoming improvements (in particular with the E-ELT) will impact on fundamental cosmology.
\end{abstract}

Keywords. Cosmology: Observations, Cosmology: theory, Techniques: spectroscopic, Methods: data analysis, Instrumentation: spectrographs

\section{Introduction}

While $\Lambda$ CDM provides the simplest viable cosmological model, fine-tuning arguments suggest that alternatives involving scalar fields may be more likely. Astrophysical measurements of nature's fundamental couplings can be used to study the properties of dark energy, either by themselves or in combination with other cosmological datasets. They complement other methods due to the large redshift lever arm. In Amendola et al. (2012) we extended Principal Component Analysis (PCA) methods and studied the feasibility of applying them to astrophysical measurements of varying couplings - whether they are detections of variations or null results.

Recent results of Webb et al. (2011) suggest that the fine-structure constant has varied over the last ten billion years, the relative variation being at the level of a few parts per million. Efforts to test this claim are ongoing, but a detailed answer may have to wait for the next generation of facilities. It is clear that observation time on these will scarce, and optimized observational strategies are essential. In Leite et al. (2014) we take some steps towards quantifying the potentialities of this method. We use current $\alpha$ measurements from VLT/UVES as a benchmark that can be extrapolated into future (simulated) datasets whose impact for dark energy characterization can be studied, specifically focusing on ESPRESSO (for the VLT), and especially in the E-ELT's highresolution spectrograph (ELT-HIRES). We summarize these results here.

Our formalism is described in Amendola et al. (2012). We will consider quintessencetype models coupled to electromagnetism, though $\mathcal{L}_{\phi F}=-1 / 4 B_{F}(\phi) F_{\mu \nu} F^{\mu \nu}$ where the gauge kinetic function $B_{F}(\phi)$ is assumed linear. Then, the evolution of $\alpha$ is linearly proportional to the field displacement, the proportionality parameter being a dimensionless coupling parameter $\zeta$. From this one can calculate the Fisher matrix using standard techniques. We consider three fiducial forms for the equation of state parameter, respectively $w_{c}(z)=-0.9, w_{s}(z)=-0.5+0.5 \tanh (z-1.5)$, and $w_{b}(z)=$

$\dagger$ We acknowledge the financial support of grant PTDC/FIS/111725/2009 from FCT (Portugal). C.J.M. is also supported by an FCT Research Professorship, contract reference IF/00064/2012, funded by FCT/MCTES (Portugal) and POPH/FSE (EC). 
Table 1. The coefficients A and B in the fitting formula 1.1, assuming 20 or 30 PCA bins in the redshift range $0<z<4$ and uncertainties $\sigma_{\alpha}$ in parts per million.

\begin{tabular}{|c|c|c|c|c|}
\hline Model & $\mathrm{A}\left(N_{\mathrm{bin}}=20\right)$ & $\mathrm{B}\left(N_{\mathrm{bin}}=20\right)$ & $\mathrm{A}\left(N_{\mathrm{bin}}=30\right)\left|\mathrm{B}\left(N_{\mathrm{bin}}=30\right)\right|$ \\
\hline Constant & 1.14 & 0.52 & 1.39 & 0.63 \\
Step & 2.10 & 0.96 & 2.53 & 1.16 \\
Bump & 1.65 & 0.75 & 2.00 & 0.91 \\
\hline
\end{tabular}

$-0.9+1.3 \exp \left(-(z-1.5)^{2} / 0.1\right)$. Phenomenologically, these describe the three qualitatively different interesting scenarios: an equation of state that remains close to a cosmological constant throughout the probed redshift range, one that evolves towards a matter-like behaviour by the highest redshifts probed, and one that has non-trivial features over a limited redshift range. In what follows we will refer to these three cases as the constant, step and bump fiducial models.

In order to systematically study possible observational strategies, it's of interest to find an analytic expression for the behaviour of the uncertainties of the best determined PCA modes described above. By exploring numbers of measurements $N_{\alpha}$ between 20 and 200, uniformly distributed in redshift up to $z=4$, and individual measurement uncertainties between $10^{-5}$ and $10^{-8}$ we find the following fitting formula for the uncertainty $\sigma_{n}$ for the $\mathrm{n}$-th best determined PCA mode

$$
\sigma_{n}=A \frac{\sigma_{\alpha}}{N_{\alpha}^{0.5}}[1+B(n-1)] .
$$

The coefficients $\mathrm{A}$ and $\mathrm{B}$ will depend on the choice of fiducial model, and also on the number of PCA bins assumed for the redshift range under consideration. Table 1 lists these coefficients for choices of 20 and 30 bins. Notice that it's useful to provide the uncertainly $\sigma_{\alpha}$ in the fitting formula in parts per million, since in that case the coefficients $A$ and $B$ are of order unity.

\section{Calibrating with VLT data}

A time normalisation can be derived from the present VLT performances. We can assume a simple (idealised) observational formula, $\sigma_{\text {sample }}^{2}=C / T$, where $C$ is a constant, $T$ is the time of observation necessary to acquire a sample of $N$ measurements and $\sigma_{\text {sample }}$ is the uncertainty in $\Delta \alpha / \alpha$ for the whole sample. This is expected to hold for a uniform sample (ie, one in which one has $N_{\alpha}$ identical objects, each of which produces a measurement with the same uncertainty $\sigma_{\alpha}$ in a given observation time). Clearly any real-data sample will not be uniform, so there will be corrections to this behaviour. The uncertainty of the sample will be given by $\sigma_{\text {sample }}^{2}=1 / \sum_{i=1}^{N} \sigma_{i}^{-2}$, and for the above simulated case with $\mathrm{N}$ measurements all with the same $\alpha$ uncertainty we trivially have $\sigma_{\text {sample }}^{2}=\sigma_{\alpha}^{2} / N$. We have used the UVES data from King (2011), complemented by observation time data provided by Michael Murphy, to build a sample to calibrate the observational formula.

The analysis of Leite et al. (2014) shows strong correlation between the number of transitions used to make one measurement $\left(N_{\lambda}\right)$ and the uncertainty corresponding to it. A simple parametrisation shows the following approximate relation

$$
\sigma_{\Delta \alpha / \alpha}=1,39 \times 10^{-4} N_{\lambda}^{-1,11} .
$$

One consequence of these properties is that the simple relation above will not strictly hold. Nevertheless, there is a simple way to correct it, which consists of allowing the 
Table 2. Number of nights needed to achieve an uncertainty $\sigma_{1}=1$ in the best-determined mode, as a function of the fiducial model and the spectrograph used.

\begin{tabular}{|c|c|c|c|}
\hline Model & Baseline & ESPRESSO & ELT-HIRES \\
\hline Constant & 6.9 & 0.7 & 0.02 \\
Step & 23.5 & 2.5 & 0.07 \\
Bump & 14.5 & 1.5 & 0.05 \\
\hline
\end{tabular}

former constant $C$ to itself depend on the number of sources. This is easy to understand: in a small sample one typically will have the best available sources; by increasing our sample we'll be adding sources which are not as good as the previous ones, and therefore the overall uncertainty in the $\alpha$ measurement will improve more slowly than in the ideal case (or alternatively one will need more telescope time.

Using standard Monte Carlo techniques we find that a good fit is provided by the linear relation

$$
C\left(N_{\alpha}\right)=0.31 N_{\alpha}+5.02 .
$$

Here the constant has been normalised such that $\sigma_{\text {sample }}$ is given in parts per million and $T$ is in nights. As a simple check, for the UVES Large Program for Testing Fundamental Physics, with about 40 nights and 16 sources, we infer from the fitting formula a value of 0.5 parts per million, consistent with the expectations of the collaboration.

\section{Future observational strategies}

We thus obtain a UVES-calibrated PCA formula

$$
\sigma_{n}=A[1+B(n-1)]\left[\frac{C\left(N_{\alpha}\right)}{T}\right]^{1 / 2},
$$

where the UVES $C(N)$ formula is given by Eq. 2.2. The most striking feature of this result is the explicit (and strong) dependence on the number of sources. Future improvements will come from a better sample selection and optimised acquisition/calibration methods and both of these are expected to significantly reduce this dependence, even eliminating if for moderately sized samples of absorbers. In the case of the ELT-HIRES, a further improvement will come from the larger collecting power.

With simple but reasonable extrapolations, Leite et al. (2014) forecast the expected changes to the UVES formula, and from this carry out an assessment of the impact of these measurements for constraining dark energy. These are summarized in Table 2. The gains achieved in going from the current baseline to ESPRESSO and ELT-HIRES are quite clear. We note that a uniform redshift cover is important in obtaining these results, and there are mild dependencies on the fiducial model and other effects. A detailed study of possible observational strategies will be presented elsewhere.

\section{References}

Amendola, L., Leite, A. C. O.., Martins, C. J. A. P., Nunes, N. J., Pedrosa, P. O. J.., \& Seganti, A. 2012, Phys. Rev. D, 86, 063515

King, J. A. 2011, UNSW PhD thesis, Searching for variations in the fine-structure constant and the proton-to-electron mass ratio using quasar absorption lines (online e-Print: arXiv:1202.6365)

Leite, A. C. O., Martins, C. J. A. P., Pedrosa, P. O. J., \& Nunes, N. J., 2014, Phys. Rev. D 90, 063519

Webb, J. K., King, J. A., Murphy, M. T., Flambaum, V. V., Carswell, R. F., \& Bainbridge, M. B. 2011, Phys. Rev. Lett., 107, 191101 